\title{
On conjugacy of GGS-groups
}

\author{
Jan Moritz Petschick \\ Communicated by Christopher W. Parker
}

\begin{abstract}
Each GGS-group on the $p$-adic tree is defined by a $(p-1)$-tuple of integers modulo $p$, called the defining tuple. It is proved that two GGS-groups are conjugate (equivalently, by a theorem of Grigorchuk and Wilson, isomorphic) in the automorphism group of the tree if and only if their defining tuples can be transformed into each other by multiplication by an integer modulo $p$ and performing certain reorderings of the tuple. Finally, the number of isomorphism classes of GGS-groups is calculated.
\end{abstract}

\section{Introduction}

Many interesting examples in group theory arise from groups acting on the $p$-regular rooted tree, $p$ being a prime number. Early constructions like the groups of Grigorchuk (cf. [6]) and of Gupta and Sidki (cf. [8]) have been generalized to the family of so-called GGS-groups (named after Grigorchuk, Gupta and Sidki), which contains important examples of finitely generated infinite torsion groups. We recall the exact definition of GGS-groups below. Each GGS-group is defined by a tuple $e \in \mathbb{F}_{p}^{p-1}$, where $\mathbb{F}_{p}$ denotes the field of order $p$. An important lemma in many proofs concerning GGS-groups and their generalizations (cf. [1, Lemma 3.4], [5, parts of Theorem 2.16]) is the following fact: Up to conjugation by a tree automorphism the first component of $e$ can be chosen to equal 1.

Here we show that this cannot be improved and that two GGS-groups defined by tuples $e$ and $e^{\prime}$ are conjugate by a tree automorphism (equivalently, by a theorem of Grigorchuk and Wilson, isomorphic) if and only if there exist $v, w \in \mathbb{F}_{p}^{\times}$such that $e_{i}^{\prime}=w \cdot e_{v \cdot i}$ for all $i \in\{1, \ldots, p-1\}$. This partially answers a question posed by Bartholdi, Grigorchuk and Šunik in [3, Chapter 12, Question 6]. Parts of the proof work for a family of generalized GGS-groups defined by Bartholdi [2] and they will be presented in this more general context.

\section{Preliminaries on rooted trees and GGS-groups}

\subsection{Rooted trees and their automorphisms}

For the rest of the paper, $\mathcal{T}$ will denote the regular $m$-adic tree, $m \geq 2$ being an integer. We will identify the vertices of $\mathcal{T}$ with elements of the free monoid $X^{*}$ 
on $X=\{1, \ldots, m\}$ in the obvious way and denote the empty word by $\epsilon$. The $n$-th layer of $\mathcal{T}$ is the set of vertices represented by words of length $n$. The operation on $X^{*}$ will be omitted from the notation, so $u v$ will always mean the concatenation of the words $u$ and $v$.

Every automorphism of $\mathcal{T}$ fixes $\epsilon$ and moreover maps the $n$-th layer to itself. The full group of automorphisms Aut $\mathcal{T}$ acts transitively on each layer. A subgroup with this property is said to be spherically transitive. The stabilizer of a word $u$ under the action of a group $G$ of automorphisms of $\mathcal{T}$ is denoted by $\operatorname{stab}_{G}(u)$ and the intersection of all stabilizers of words of length $n$ is called the $n$-th level stabilizer, denoted $\operatorname{Stab}_{G}(n)$.

Let $f$ be an automorphism of $\mathcal{J}$ and let $u, v$ be words. Since layers are invariant under $f$, the equation

$$
f(u v)=\left.f(u) f\right|_{u}(v)
$$

defines a unique automorphism $\left.f\right|_{u}$ of $\mathcal{T}$ called the section of $f$ at $u$. This automorphism can be thought of as the automorphism induced by $f$ by identifying the rooted subtrees of $\mathcal{T}$ at the vertices $u$ and $f(u)$ with the tree $\mathcal{T}$. If $G$ is a group of automorphisms, $\left.G\right|_{u}$ will denote the set of all sections of group elements at $u$. The restriction of $\left.f\right|_{u}$ to the set of words of length 1 is a permutation of $X$ and it will be written $\left.f\right|^{u}$.

The following holds for any words $u, v$ and automorphisms $f, g$ :

$$
\begin{aligned}
\left.\left(\left.f\right|_{u}\right)\right|_{v} & =\left.f\right|_{u v}, \\
\left.(f g)\right|_{u} & =\left.\left.f\right|_{g(u)} g\right|_{u}, \\
\left.f^{-1}\right|_{u} & =\left(\left.f\right|_{f^{-1}(u)}\right)^{-1} .
\end{aligned}
$$

The same equations hold for the restrictions $\left.f\right|^{u}$, so the action of $f$ on any word $x_{1} \ldots x_{n}$ of length $n$ is given by

$$
\begin{aligned}
f\left(x_{1} \ldots x_{n}\right) & =\left.\left.f\right|^{\epsilon}\left(x_{1}\right) f\right|_{x_{1}}\left(x_{2} \ldots x_{n}\right) \\
& =\left.\left.\left.f\right|^{\epsilon}\left(x_{1}\right) f\right|^{x_{1}}\left(x_{2}\right) \ldots f\right|^{x_{1} \ldots x_{n-1}}\left(x_{n}\right) .
\end{aligned}
$$

Hence an automorphism $f$ is completely described by the map $\left.u \mapsto f\right|^{u}, u \in X^{*}$, called the portrait of $f$. The automorphism induced by a permutation $\sigma$ of $X$ has constant portrait $u \mapsto \sigma$ and is denoted by $\kappa(\sigma)$. An automorphism $f$ for which $\left.f\right|^{u}$ is trivial for all $u \neq \epsilon$ is called a rooted automorphism.

Given a set $f_{1}, \ldots, f_{m}$ of $m$ automorphisms of $\mathcal{T}$, a new automorphism $f$ can be defined by

$$
\left.f\right|_{x}=f_{x} \text { and } f(x)=x
$$

for all $x \in X$. This automorphism will be denoted by $\left(f_{1}, \ldots, f_{m}\right)$. 


\subsection{GGS-groups}

Let $\sigma_{1}, \ldots, \sigma_{m-1}$ be permutations of $X$ such that the subgroup generated by them acts transitively on $X$. Now let $a_{i}$ (for $i \in\{1, \ldots, m-1\}$ ) be the automorphism of $\mathcal{T}$ that permutes the rooted subtrees of $\mathcal{T}$ whose roots are the words of length 1 according to the permutation $\sigma_{i}$; thus it effects the permutation $\sigma_{i}$ on the first letter of each non-empty word. A directed automorphism is defined by

$$
b=\left(a_{1}, \ldots, a_{m-1}, b\right) .
$$

The subgroup $G_{\Sigma}$ generated by the set $\left\{a_{i}: 1 \leq i<m\right\} \cup\{b\}$ is called the GGS-group with defining tuple $\Sigma=\left(\sigma_{1}, \ldots, \sigma_{m-1}\right)$. We say that $b$ is the defining directed automorphism of $G_{\Sigma}$. The subgroup of Aut $\mathcal{T}$ generated by the rooted automorphisms $\left\{a_{i}: 1 \leq i<m\right\}$, called the root group, will be denoted by $A$. Since $b$ acts trivially on the first layer, $\left.A \cong G_{\Sigma}\right|^{\epsilon}=\left\langle\sigma_{i}: 1 \leq i<m\right\rangle$.

As an important special case we obtain the classical GGS-groups, where $m=p$ is an odd prime and every $\sigma_{i}$ is the $e_{i}$-th power of the cyclic permutation $(1 \ldots p)$, with $e_{i} \neq 0$ for at least one $i$. In this case the defining tuple $\left(\sigma_{1}, \ldots, \sigma_{p-1}\right)$ is simply written $e=\left(e_{1}, \ldots, e_{p-1}\right)$.

We will restrict our attention to GGS-groups such that the root group is abelian. This implies that $|A|=m$ and for all $i, j \in X$ there is a unique element $a_{(i \mapsto j)} \in A$ with $a_{(i \mapsto j)}(i)=j$. The conjugate $b^{a_{(i \mapsto j)}}$ of $b$, denoted by $b_{(i, j)}$, satisfies

$$
\left.b_{(i, j)}\right|_{i}=\left.b\right|_{j}= \begin{cases}a_{j}, & \text { for } j \neq m, \\ b, & \text { for } j=m .\end{cases}
$$

As an abbreviation we will write $b_{i}$ for $b_{(m, i)}$.

\subsection{Properties of GGS-groups}

Every GGS-group $G$ is strongly self-similar, i.e. it has the following property: For each word $u$ the groups

$$
\left.G\right|_{u}=\left.\operatorname{stab}_{G}(u)\right|_{u}=G
$$

are equal. We recall briefly the proof. The statement is obvious for $\epsilon$. Assume that the statement is true for all words of length smaller than $n$ and let $u=u_{1} \ldots u_{n}$ be of length $n$. Set $\tilde{u}=u_{1} \ldots u_{n-1}$. Thus there exists an element $g_{i} \in \operatorname{stab}_{G}(\tilde{u})$ such that $\left.g_{i}\right|_{\tilde{u}}=b_{\left(u_{n}, i\right)}$ and therefore $\left.g_{i}\right|_{u}=\left.b_{\left(u_{n}, i\right)}\right|_{u_{n}}=\left.b\right|_{i}$ for all $i \in\{1, \ldots, m\}$. The sections of $b$ generate $G$, so $\left.\operatorname{stab}_{G}(u)\right|_{u} \geq G$ holds. Conversely, since $G$ is generated by $A \cup\{b\}$ and all sections of these generators are again generators, $\left.G\right|_{u} \leq G$. This implies the property for $u$. 
Furthermore, GGS-groups are spherically transitive. This is easily seen as $A$ acts transitively on $X$ by definition and (by the above paragraph)

$$
A=\left.G\right|^{\epsilon}=\left.\left(\left.G\right|_{u}\right)\right|^{\epsilon}=\left.G\right|^{u}
$$

holds for any word $u$.

Since $\left.a_{i}\right|^{u}=1$ holds for all $u \neq \epsilon$ and $i \in\{1, \ldots, m-1\}$, it follows that $a_{i}$ is the trivial automorphism if and only if $\sigma_{i}=1$ and $A \cap \operatorname{Stab}_{G}(1)=\{1\}$. Therefore the stabilizer of the first layer is generated by the set $b^{A}=\left\{b_{i}: 1 \leq i<m\right\}$ and $G=A \ltimes \operatorname{Stab}_{G}(1)$.

Finally, GGS-groups are contracting with respect to word length in the alphabet $A \cup b^{A}$, i.e. for any element $g$ of a GGS-group $G$ and a representation of $g$ as a word of length $\geq 2$, every non-trivial section of $g$ has a representation of smaller length.

Let $a b_{w_{1}}^{v_{1}} \ldots b_{w_{n}}^{v_{n}}$ be a word in the alphabet $A \cup b^{A}$, with $a \in A, b_{w_{i}} \in b^{A}$ and $b_{w_{i}} \neq b_{w_{i+1}}$ for $i \in\{1, \ldots, n-1\}$ and $n \geq 1$, representing an element $g$. Let $x$ be any letter of $X$. Since $b_{i}$ fixes $x$ for all $i$, a representation of $\left.g\right|_{x}$ is

$$
\left.a\right|_{x}\left(\left.b_{w_{1}}\right|_{x}\right)^{v_{1}} \ldots\left(\left.b_{w_{n}}\right|_{x}\right)^{v_{n}} .
$$

Let $b_{w_{i}}, b_{w_{j}}$ be two adjacent syllables, i.e. $i+1=j$. Since $A$ acts regularly on $X$, $b_{w_{i}} \neq b_{w_{j}}$ implies that at least one section at $x$ is an element of $A$, since only one section of $b$ at $x$ is not. We collect all the resulting elements of $A$ and obtain a word with fewer syllables representing $\left.g\right|_{x}$.

\section{Conjugation of GGS-groups}

We give a proof of the following well-known lemma for the reader's convenience:

Lemma 3.1. Let $G$ be the GGS-group defined by $\Sigma=\left(\sigma_{1}, \ldots, \sigma_{m-1}\right)$ on the $m$-adic tree $\mathcal{T}, n$ a number coprime to $m$ and $\tau$ a permutation of $X$ that fixes m. Then:

(1) The GGS-group defined by $\Sigma^{n}=\left(\sigma_{1}^{n}, \ldots, \sigma_{m-1}^{n}\right)$ is equal to $G$.

(2) $G^{\kappa(\tau)}$ is again a GGS-group, defined by $\left(\sigma_{\tau(1)}^{\tau}, \ldots, \sigma_{\tau(m-1)}^{\tau}\right)$.

Proof. (1) Since $n$ is coprime to $m=|A|$, both root groups are equal. For the defining directed automorphism $b$ of $G_{\Sigma}$,

$$
b^{n}=\left(a_{1}, \ldots, a_{m-1}, b\right)^{n}=\left(a_{1}^{n}, \ldots, a_{m-1}^{n}, b^{n}\right)
$$

holds. But this is the defining equation for the defining directed automorphism of $G_{\Sigma^{n}}$. 
(2) This follows directly by calculating $b^{\kappa(\tau)}$. For $i \in X$ the rules for the calculation of the section at $i$ give

$$
\left.b^{\kappa(\tau)}\right|_{i}=\left(\left.b\right|_{\tau(i)}\right)^{\kappa(\tau)}= \begin{cases}a_{\tau(i)}^{\kappa(\tau)}, & \text { for } i \neq m, \\ b^{\kappa(\tau)}, & \text { for } i=m,\end{cases}
$$

and since $b$ and thus its conjugates stabilize the first layer this fully defines

$$
b^{\kappa(\tau)}=\left(a_{\tau(1)}^{\kappa(\tau)}, \ldots, a_{\tau(m-1)}^{\kappa(\tau)}, b^{\kappa(\tau)}\right) .
$$

Since the set $\left\{\left.a_{i}\right|^{\epsilon}: i=1, \ldots, m-1\right\}$ generates a transitive abelian subgroup of $S_{m}$, the set of conjugates of the form $\left.a_{i}^{\kappa(\tau)}\right|^{\epsilon}=\left(\left.a_{i}\right|^{\epsilon}\right)^{\tau}$ does so as well.

The lemma above gives a sufficient condition for GGS-groups defined by different tuples to be conjugate. We shall prove that this condition is necessary. In order to do this, we must make some further preparations.

If $G$ and $G^{\prime}$ are two conjugate GGS-groups in Aut $\mathcal{T}$, then the groups $H, H^{\prime}$ generated by the entries in the defining tuples are conjugate in $\mathrm{S}_{m}$, since

$$
H^{\prime}=\left.G^{\prime}\right|^{\epsilon}=\left.G^{f}\right|^{\epsilon}=\left(\left.G\right|^{\epsilon}\right)^{\left.f\right|^{\epsilon}}=H^{\left.f\right|^{\epsilon}}
$$

holds if $G^{f}=G^{\prime}$ for an automorphism $f$.

Lemma 3.2. Let $G, G^{\prime}$ be GGS-groups, and let $f$ be a tree automorphism such that $G^{f}=G^{\prime}$. Then for any word $u$ and $i, j \in\{1, \ldots, m\}$, the cosets $\left.G f\right|_{u i}$ and $\left.G f\right|_{u j}$ in Aut $\mathcal{T}$ are equal.

Proof. Since $G^{\prime}=\left.\operatorname{stab}_{G^{\prime}}(u)\right|_{u}$, there is an element $h=g^{f} \in \operatorname{stab}_{G^{\prime}}(u)$ with $\left.h\right|_{u}=a_{(i \mapsto j)}$ and $g \in \operatorname{stab}_{G}(f(u))$. We then calculate

$$
g^{f}(u i)=\left.g^{f}(u) g^{f}\right|_{u}(i)=u j
$$

and substitute into

$$
\begin{aligned}
1=\left.a_{(i \mapsto j)}\right|_{i} & =\left.g^{f}\right|_{u i}=\left.\left.\left.f^{-1}\right|_{g(f(u i))} g\right|_{f(u i)} f\right|_{u i} \\
& =\left.\left.\left(\left.f\right|_{g f(u i)}\right)^{-1} g\right|_{f(u i)} f\right|_{u i}=\left.\left.\left(\left.f\right|_{u j}\right)^{-1} g\right|_{f(u i)} f\right|_{u i} .
\end{aligned}
$$

Since $\left.\left.g\right|_{f(u i)} \in G\right|_{f(u i)}=G$, this establishes the assertion.

Using this lemma, we shall find a vertex for which conjugation by the section of $f$ maps $G$ onto $G^{\prime}$ and for which there is a simple relation between the defining directed automorphisms. But first, we need a more general lemma. 
Lemma 3.3. Let $G$ be a group of tree automorphisms satisfying $G=\left.\operatorname{stab}_{G}(u)\right|_{u}$ for all words u (e.g. a strongly self-similar or in particular a GGS-group) and $f$ a tree automorphism such that $G^{f}$ again satisfies $G^{f}=\left.\operatorname{stab}_{G^{f}}(u)\right|_{u}$ for all words $u$. Then $G^{\left.f\right|_{u}} \leq G^{f}$.

Proof. Let $g \in G$ be arbitrary and $h \in \operatorname{stab}_{G}(f(u))$ such that $\left.h\right|_{f(u)}=g$. Then

$$
g^{\left.f\right|_{u}}=\left(\left.h\right|_{f(u)}\right)^{\left.f\right|_{u}}=\left.\left.\left(h^{f}\right)\right|_{u} \in \operatorname{stab}_{G^{f}}(u)\right|_{u}=G^{f} .
$$

Lemma 3.4. Let $G$ be a GGS-group and let $f$ be a tree automorphism such that $G^{\prime}=G^{f}$ is again a GGS-group. Then there exist a positive integer $k$ and an element $w \in\{1, \ldots, m-1\}$ such that $G^{\left.f\right|_{m^{k}}}=G^{f}$ and $b^{\prime}=\left(b^{w}\right)^{\left.f\right|_{m^{k}}}$ hold, where $b$ and $b^{\prime}$ are the defining directed automorphisms of $G$ and $G^{\prime}$, respectively.

Proof. For $i \in\{1, \ldots, m-1\}$ write $a_{i}$, resp. $a_{i}^{\prime}$, for the sections at the words of length 1 of the defining directed automorphism of $G$, resp. $G^{\prime}$. Let

$$
h_{0}=\prod_{j=1}^{n} b_{v_{j}}^{k_{j}} \in \operatorname{stab}_{G}(1)
$$

(where $n, k_{j}$ are positive integers and $v_{j} \in X$ for $1 \leq j, i \leq n$ ) be the element satisfying

$$
h_{0}^{f}=b^{\prime} .
$$

As a first step, we will show that all generators $a_{1}^{\prime}, \ldots, a_{m-1}^{\prime}, b^{\prime}$ are in $G^{f \mid m}$. Together with the statement of the last lemma this gives

$$
G^{\left.f\right|_{m}}=G^{f} .
$$

Consider

$$
b^{\prime}=\left.b^{\prime}\right|_{m}=\left.\prod_{j=1}^{n}\left(b_{v_{j}}^{k_{j}}\right)^{f}\right|_{m}=\prod_{j=1}^{n}\left(\left.b_{v_{j}}^{k_{j}}\right|_{f(m)}\right)^{\left.f\right|_{m}} \in G^{\left.f\right|_{m}}
$$

and

$$
a_{i}^{\prime}=\left.b^{\prime}\right|_{i}=\left.\prod_{j=1}^{n}\left(b_{v_{j}}^{k_{j}}\right)^{f}\right|_{i}=\prod_{j=1}^{n}\left(\left.b_{v_{j}}^{k_{j}}\right|_{f(i)}\right)^{\left.f\right|_{i}} \in G^{\left.f\right|_{i}}
$$

with $i \in\{1, \ldots, m-1\}$. Write

$$
h_{1}=\left.\prod_{j=1}^{n}\left(b_{v_{j}}^{k_{j}}\right)\right|_{f(m)}
$$


for the element satisfying $h_{1}^{\left.f\right|_{m}}=b^{\prime}$. By Lemma 3.2, $G^{\left.f\right|_{i}}=G^{\left.f\right|_{m}}$ for any $i$. This proves that $G^{f}=G^{\left.f\right|_{m}}$. Since $\left.\left(\left.f\right|_{m}\right)\right|_{m}=\left.f\right|_{m m}$ holds, this procedure can be iterated to show that $G^{\left.f\right|_{m} l}=G^{f}$ for any positive integer $l$. Now we look at the length of the word representing the element $h_{l}$ of $G$ satisfying

$$
h_{l}^{\left.f\right|_{m} l}=b^{\prime} .
$$

By the formula above, it is represented by the following section of $h_{0}$ :

$$
\left.\prod_{j=1}^{n}\left(b_{v_{j}}^{k_{j}}\right)\right|_{f(m)^{l}}
$$

Since GGS-groups are contracting, there is a positive integer $k$ such that the length of $h_{k}$ is 1 , i.e. there are $v, w \in X$ such that

$$
\left(b_{v}^{w}\right)^{\left.f\right|_{m^{k}}}=b^{\prime} .
$$

But taking a section at $m$ gives

$$
\left(\left.b_{v}^{w}\right|_{\left.f\right|_{m^{k}}(m)}\right)^{\left.f\right|_{m^{k+1}}}=b^{\prime} \in \operatorname{stab}_{G^{f}}(1) .
$$

Now $\left.b_{v}^{w}\right|_{\left.f\right|_{m^{k}}(m)}$ is either equal to some $a \in A$ or to $b^{w}$, since $b_{v}=b^{a}$ for an $a \in A$. Since $b^{\prime}$ stabilizes the first layer, $\left.b_{v}^{w}\right|_{\left.f\right|_{m k}(m)}$ must do so as well and therefore it must be equal to $b^{w}$. Finally, we obtain

$$
\left(b^{w}\right)^{\left.f\right|_{m k+1}}=b^{\prime} .
$$

Theorem 3.5. Let $G$ be a GGS-group defined by $\left(\sigma_{1}, \ldots, \sigma_{m-1}\right)$ and $f$ a tree automorphism such that $G^{f}$ is a GGS-group defined by $\left(\sigma_{1}^{\prime}, \ldots, \sigma_{m-1}^{\prime}\right)$. Then there are two permutations $\tau, \rho$ that fix $m$ and a positive integer $n$ coprime to $m$ such that for all $i \in\{1, \ldots, m-1\}$,

$$
\sigma_{i}^{\prime}=\left(\sigma_{\tau(i)}^{n}\right)^{\rho} .
$$

Additionally, $\tau=\left.f\right|^{\epsilon}$.

Proof. By Lemma 3.4 we can, without loss of generality, reduce to $b^{\prime}=\left(b^{n}\right)^{f}$. Define $f_{s}$ by

$$
f_{s}=\kappa\left(\left.f\right|^{\epsilon}\right)^{-1} f .
$$

Then $f_{s}$ stabilizes the first layer, since $\left.f_{s}\right|^{\epsilon}=\left.\left.\left(\kappa\left(\left.f\right|^{\epsilon}\right)^{-1}\right)\right|^{\epsilon} f\right|^{\epsilon}=1$. The equation

$$
\left.\begin{array}{ll}
a_{i}^{\prime}, & \text { for } i \neq m, \\
b^{\prime}, & \text { for } i=m,
\end{array}\right\}=\left.b^{\prime}\right|_{i}=\left(\left.b^{n}\right|_{f \mid \epsilon(i)}\right)^{\left.f\right|_{i}}= \begin{cases}\left(a_{f \mid \epsilon(i)}^{n}\right)^{\left.f\right|_{i},} & \text { for }\left.f\right|^{\epsilon}(i) \neq m, \\
\left(b^{n}\right)^{\left.f\right|_{i},}, & \text { for }\left.f\right|^{\epsilon}(i)=m,\end{cases}
$$


shows that $\left.f\right|^{\epsilon}(m)=m$, since $\left(b^{n}\right)^{\left.f\right|_{i}}$ is not a rooted automorphism. If it were rooted, it would be trivial, since $\left.\left.\left(b^{n}\right)^{f \mid}\right|_{i}\right|^{\epsilon}=\left(b^{n} \mid{ }^{\epsilon}\right)^{\left.f\right|^{i}}=1$. But $b^{n}$ is not trivial. Therefore, by Lemma 3.1, $G^{\kappa\left(\left.f\right|^{\epsilon}\right)}$ is again a GGS-group, whose defining tuple has entries of the form $\left(\left.\sigma_{f}\right|_{(i)}\right)^{\left.f\right|^{\epsilon}}$. By replacing $G$ with $G^{\kappa\left(\left.f\right|^{\epsilon}\right)}$, we can reduce to the case $f \in \operatorname{Stab}_{G}(1)$.

Now for brevity write $f=f_{s} \in \operatorname{Stab}_{G}(1)$. By the last equation,

$$
\left(a_{i}^{n}\right)^{\left.f\right|_{i}}=a_{i}^{\prime}
$$

for all $i \neq m$. By Lemma 3.2 we have $\left.G f\right|_{i}=\left.G f\right|_{m}$ for each $i \in X$. This gives

$$
\left.\left.\left.G\right|^{\epsilon} f\right|_{i}\right|^{\epsilon}=\left.\left.A\right|^{\epsilon} f\right|^{i}=\left.\left.A\right|^{\epsilon} f\right|^{m}=\left.\left.\left.G\right|^{\epsilon} f\right|_{m}\right|^{\epsilon} .
$$

Further, $A$ is abelian by definition, so

$$
\left(a_{i}^{n}\right)^{f \mid m}=\left(a_{i}^{n}\right)^{\left.f\right|_{i}}=a_{i}^{\prime}
$$

holds for all $i \neq m$. Together with the equation for the constant part $\kappa\left(\left.f\right|^{\epsilon}\right)$ this gives the general formula. Since only the rooted part $\left.f\right|^{\epsilon}$ contributes to the permutation reordering the entries of the defining tuple, and since by the first paragraph this permutation is equal to $\left.f\right|^{\epsilon}$, the additional assertion is proven as well.

Corollary 3.6. Two classical GGS-groups are conjugate if and only if there exist $v, w \in \mathbb{F}_{p}^{\times}$such that their defining tuples $\left(e_{1}, \ldots, e_{p-1}\right)$ and $\left(e_{1}^{\prime}, \ldots, e_{p-1}^{\prime}\right)$ satisfy

$$
e_{i}^{\prime}=w \cdot e_{v \cdot i}
$$

for all $i$.

Proof. We recall that for a classical GGS-group we have $\sigma_{i} \in\langle(1 \ldots p)\rangle$ for all $i \neq m$ and $m=p$ a prime number. We will write $c=(1 \ldots p)$ from here on. Assume that a tree automorphism $f$ conjugates a classical GGS-group $G$ into another classical GGS-group $G^{\prime}$. By Theorem 3.5 there is a permutation $\rho$ of $X$ such that for all $i \in\{1, \ldots, m-1\}$,

$$
\sigma_{i}^{\prime}=\left(\sigma_{\left.f\right|^{\epsilon}(i)}^{n}\right)^{\rho} .
$$

Since

$$
\langle c\rangle=\left.A^{f}\right|^{\epsilon}=\left(\left.A\right|^{\epsilon}\right)^{\left.f\right|^{\epsilon}}=\langle c\rangle^{\left.f\right|^{\epsilon}},
$$

it follows that $\left.f\right|^{\epsilon}$ normalizes $\langle c\rangle$, and as we have seen in the first step of the proof of Theorem 3.5, stabilizes $p$. Since $A=A^{\prime}$, the permutation $\rho$ must also normalize $\langle c\rangle$. It is well known that

$$
\mathrm{N}_{\mathrm{S}_{p}}(\langle c\rangle) \cong \operatorname{Aut}(\langle c\rangle) \ltimes\langle c\rangle .
$$

Conjugation of the $\sigma_{i}$ by a fixed element of $\mathrm{N}_{S_{p}}(\langle c\rangle)$ will result in raising all $\sigma_{i}$ to some power. Therefore the permutation $\rho$ can be omitted. Both remaining actions 
on the classical defining tuple (which is represented by the exponent of $c$ in each entry of the usual defining tuple) can be described by multiplication in $\mathbb{F}_{p}$ :

- Raising all entries to the $w$-th power corresponds to multiplication by $w$.

- Reordering the entries by a member of $\operatorname{Stab}_{S_{p}}(p) \cap \mathrm{N}_{S_{p}}(\langle c\rangle)$ can be represented by multiplication of the index of an entry by an element of $\mathbb{F}_{p}^{\times}$, since $\operatorname{Stab}_{\mathrm{S}_{p}}(p) \cap \mathrm{N}_{\mathrm{S}_{p}}(\langle c\rangle) \cong \mathbb{F}_{p}^{\times}$.

This proves the "only if" part. The "if" part results from Lemma 3.1.

\section{Number of isomorphism classes of classical GGS-groups}

Using Corollary 3.6, we can calculate the number of classical GGS-groups up to conjugacy. We reformulate the corollary: Two classical GGS-groups are conjugate if and only if their defining tuples lie in the same orbit of the action

$$
\begin{aligned}
\kappa:\left(\mathbb{F}_{p}^{\times} \times \mathbb{F}_{p}^{\times}\right) & \rightarrow \mathrm{S}\left(\mathbb{F}_{p}^{p-1}\right), \\
(v, w) & \mapsto\left(e_{1}, \ldots, e_{p-1}\right) \mapsto\left(w e_{v}, \ldots, w e_{v(p-1)}\right) .
\end{aligned}
$$

Moreover, each element of $\mathbb{F}_{p}^{p-1} \backslash\{0\}$ defines a classical GGS-group. The number of classical GGS-groups up to conjugacy is thus one less than the number of orbits of $\kappa$.

We fix a generator $g$ of $\mathbb{F}_{p}^{\times}$. Let $g^{j}$ and $i$ be arbitrary elements of $\mathbb{F}_{p}^{\times}$(the reason for this unusual specification will become apparent soon) and let

$$
e=\left(e_{1}, \ldots, e_{p-1}\right) \in \mathbb{F}_{p}^{p-1}
$$

be a fixed point of $\kappa\left(g^{j}, i\right)$.

The element $g^{j}$ permutes the indices and thus is a product of cycles. The order of this permutation is

$$
l=\frac{p-1}{(j, p-1)},
$$

with $(j, p-1)$ being the greatest common divisor of $j$ and $p-1$, equal to the order of $g^{j}$. The permutation decomposes into cycles of the same length, since $\left(g^{j}\right)^{y} x=x$ holds independently of $x$ for $y=l$. Each cycle must have length $l$ and the number of cycles is $a=(j, p-1)$. Let $\left(b_{0}, \ldots, b_{l-1}\right)$ be such a cycle. Since $e$ is a fixed point,

$$
e_{b_{0}}=i \cdot e_{b_{1}}=\cdots=i^{l-1} \cdot e_{b_{l-1}}=i^{l} \cdot e_{b_{0}}
$$

holds. If the order of $i$ as an element of $\mathbb{F}_{p}^{\times}$does not divide $l$, the only possibility for this to hold is $e_{b_{0}}=\cdots=e_{b_{l-1}}=0$. Since the cycle was arbitrary, the only fixed point in this case is 0 . 
If otherwise $i^{l}=1$, the elements $e_{b_{1}}, \ldots, e_{b_{l-1}}$ are determined by $e_{b_{0}}$. For every cycle of the permutation describing multiplication by $g^{j}$, one entry can freely be chosen. Thus, in this case the number of fixed points is $p^{a}$. By Burnside's Lemma on the number of orbits we obtain:

Theorem 4.1. The number $\#_{\mathrm{GGS}}(p)$ of classical GGS-groups up to conjugacy for a fixed odd prime $p$ is

$$
\#_{\mathrm{GGS}}(p)=-1+\frac{1}{(p-1)^{2}} \sum_{j \in \mathbb{F}_{p}^{\times}} \sum_{i \in \mathbb{F}_{p}^{\times}} \begin{cases}1, & \text { for } \operatorname{ord}_{\mathbb{F}_{p}^{\times}}(i) \nmid \frac{p-1}{(j, p-1)}, \\ p^{(j, p-1)}, & \text { for } \operatorname{ord}_{\mathbb{F}_{p}^{\times}}(i) \mid \frac{p-1}{(j, p-1)}\end{cases}
$$

Corollary 4.2. The growth rate of $\#_{\mathrm{GGS}}(p)$ is $p^{p-3}$.

Proof. We have $(j, p-1)=p-1$ if and only if $j=p-1$. But then $\operatorname{ord}_{\mathbb{F}_{p}^{\times}}(i)$ must be 1 to divide $\frac{p-1}{(j, p-1)}$. Thus

$$
\#_{\mathrm{GGS}}(p)=\frac{1}{(p-1)^{2}}\left(p^{p-1}+\text { monomials of lesser order }\right) .
$$

As a last result we will extend our results by replacing "conjugacy" by "isomorphism", using a result of Grigorchuk and Wilson showing that these properties coincide for classical GGS-groups. To apply the theorem, we need some further properties of GGS-groups.

The rigid stabilizer $\operatorname{rst}_{G}(u)$ of a word $u$ is the set of automorphisms of a subgroup $G$ of Aut $\mathcal{J}$ fixing all words not starting with $u$. The $n$-th rigid layer stabilizer $\operatorname{Rst}_{G}(n)$ is the product of all rigid stabilizers of words of length $n$.

A group of tree automorphisms that is spherically transitive and has the property that all rigid layer stabilizers have finite index is called a branch group. By a result of Fernández-Alcober and Zugadi-Reizabal [5], all classical GGS-groups whose defining tuple is not constant are branch groups. The GGS-groups with constant defining tuple however are not branch groups, cf. [4].

There are two other interesting properties, commonly denoted by $(*)$ and $(* *)$ :

(*) $\left.\operatorname{stab}_{G}(u)\right|^{u}$ is cyclic for every word $u$.

(**) For any words $u, v$ that are not prefixes of each other and any $u^{\prime} \neq u$ starting

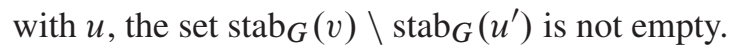

Any classical GGS-group obviously satisfies (*) by the definition of "classical". In the next lemma, we turn our attention to property $(* *)$. Two particular cases of the following lemma have already been proved, one in [7, Lemma 6], where the above properties were first defined, and another in [9, Proposition 3.7]. 
Lemma 4.3. Every classical GGS-group G satisfies (**).

Proof. By strong self-similarity, the smallest common prefix of $u$ and $v$ is the empty word. First we consider the case $v=p \ldots p$ and $u^{\prime}$ of length greater than 1 and starting with the letter $j \neq p$. Let $\left(e_{1}, \ldots, e_{p-1}\right)$ be the defining tuple. If $e_{j} \neq 0$, the directed automorphism is in $\operatorname{stab}_{G}(v) \backslash \operatorname{stab}_{G}\left(u^{\prime}\right)$. Otherwise we look at $b b_{j}$ : Either $\left.b_{(p, j)}\right|_{j}=a^{e_{2 j}}=1$ or $b b_{(p, j)} \in \operatorname{stab}_{G}(v) \backslash \operatorname{stab}_{G}\left(u^{\prime}\right)$ holds, since (recalling that $a$ is the cyclic permutation, since $G$ is classical) we have

$$
\left.b b_{(p, j)}\right|_{p}=\left.\left.b\right|_{p} b^{a^{j}}\right|_{p}=b a^{e_{j}}=b \text { and }\left.b b_{(p, j)}\right|_{j}=a^{e_{j}} a^{e_{2 j}} .
$$

In the first case, we look at $b b_{(p, 2 j)}$ and so on. Since there is an entry unequal to 0 in the defining tuple, after some steps an element of $\operatorname{stab}_{G}(v) \backslash \operatorname{stab}_{G}\left(u^{\prime}\right)$ is found. Now let $v$ be arbitrary and $g \in G$ such that $g(v)=p \ldots p$. Since $u^{\prime}$ is at least of length 2 and starts with a letter $u_{1}^{\prime}$ different from the first letter $v_{1}$ of $v$, we have $\left.g\right|^{\epsilon}\left(v_{1}\right)=p \neq\left. g\right|^{\epsilon}\left(u_{1}^{\prime}\right)$ and thus $g\left(u^{\prime}\right)$ does not begin with $p$. Choose some $h \in \operatorname{stab}_{G}(p \ldots p) \backslash \operatorname{stab}_{G}\left(g\left(u^{\prime}\right)\right)$. The existence of such an element was proven in the last step. Then $h^{g} \in \operatorname{stab}_{G}(v) \backslash \operatorname{stab}_{G}\left(u^{\prime}\right)$.

Finally, we state a result of Grigorchuk and Wilson [7]:

Theorem 4.4 (Grigorchuk-Wilson). Let $G, H$ be branch groups in Aut $\mathcal{T}$ and suppose that $G$ has properties $(*)$ and $(* *)$. If there is an isomorphism $\varphi: G \rightarrow H$, there is a tree automorphism $f$ such that $\varphi$ is induced by conjugation with $f$.

This allows to improve the statements of our Theorems 3.6 and 4.1 by replacing "conjugate" by "isomorphic", since for all classical GGS-groups not defined by a constant tuple the properties coincide by Theorem 4.4. But those defined by a constant tuple are all conjugate by Lemma 3.1 and not branch groups (again, cf. [4]) and thus not isomorphic to a classical GGS-group defined by a non-constant tuple.

We repeat our improved theorems as corollaries:

Corollary 4.5. Two classical GGS-groups are isomorphic if and only if there exist $v, w \in \mathbb{F}_{p}^{\times}$such that their defining tuples $\left(e_{1}, \ldots, e_{p-1}\right)$ and $\left(e_{1}^{\prime}, \ldots, e_{p-1}^{\prime}\right)$ satisfy

$$
e_{x}^{\prime}=w \cdot e_{v \cdot x}
$$

for all $x$.

Corollary 4.6. The number $\#_{\mathrm{GGS}}(p)$ of classical GGS-groups up to isomorphism for a fixed odd prime $p$ is

$$
\#_{\mathrm{GGS}}(p)=-1+\frac{1}{(p-1)^{2}} \sum_{j \in \mathbb{F}_{p}^{\times}} \sum_{i \in \mathbb{F}_{p}^{\times}} \begin{cases}1, & \text { for } \operatorname{ord}_{\mathbb{F}_{p}^{\times}}(i) \nmid \frac{p-1}{(j, p-1)}, \\ p^{(j, p-1)}, & \text { for } \operatorname{ord}_{\mathbb{F}_{p}^{\times}}(i) \mid \frac{p-1}{(j, p-1)}\end{cases}
$$


Acknowledgments. The results of this paper were developed as part of the authors Diploma Thesis at the University of Leipzig supervised by Professor John Wilson, for whose helpful comments and insights the author is very grateful.

\section{Bibliography}

[1] T. Alexoudas, B. Klopsch and A. Thillaisundaram, Maximal subgroups of multi-edge spinal groups, Groups Geom. Dyn. 10 (2016), no. 2, 619-648.

[2] L. Bartholdi, Croissance de groupes agissant sur des arbres, Ph.D. thesis, Université de Genève, 2000

[3] L. Bartholdi, R. I. Grigorchuk and Z. Šunik, Branch groups, in: Handbook of Algebra. Vol. 3, Elsevier/North-Holland, Amsterdam (2003), 989-1112.

[4] G. A. Fernández-Alcober, A. Garrido and J. Uria-Albizuri, On the congruence subgroup property for GGS-groups, Proc. Amer. Math. Soc. 145 (2017), no. 8, 3311-3322.

[5] G. A. Fernández-Alcober and A. Zugadi-Reizabal, GGS-Groups: Order of congruence quotients and Hausdorff dimension, Trans. Amer. Math. Soc. 366 (2014), no. 4, 1993-2017.

[6] R. I. Grigorchuk, On Burnside's problem on periodic groups, Funktsional. Anal. $i$ Prilozhen. 14 (1980), no. 1, 53-54.

[7] R. I. Grigorchuk and J.S. Wilson, The uniqueness of the actions of certain branch groups on rooted trees, Geom. Dedicata 100 (2003), 103-116.

[8] N. D. Gupta and S. N. Sidki, On the Burnside problem for periodic groups, Math. Z. 182 (1983), 385-388.

[9] B. Klopsch und A. Thillaisundaram, Maximal subgroups and irreducible representations of generalised multi-edge spinal groups, preprint (2016), https://arxiv . org/abs/1605.02907.

Received June 27, 2018; revised December 7, 2018.

\section{Author information}

Jan Moritz Petschick, Mathematisch-Naturwissenschaftliche Fakultät, Heinrich-Heine-Universität Düsseldorf, Universitätsstraße 1, Düsseldorf, Germany. E-mail: jan.petschick@hhu.de 